\title{
Skills training reduced sexual risk behaviours in homeless men with mental illness
}

\author{
Susser E, Valencia E, Berkman A, et al. Human immunodeficiency virus sexual risk reduction in homeless men with mental illness. \\ Arch Gen Psychiatry 1998 Mar;55:266-72.
}

\section{Question}

Can a skills training intervention reduce sexual risk behaviours in homeless men who have mental illness?

\section{Design}

Randomised controlled trial with 6 and 18 months follow up.

\section{Setting}

200 bed municipal men's shelter in New York City, New York, USA.

\section{Patients}

97 men attending a psychiatric outreach programme. 59 of the men were sexually active at baseline (defined as having had vaginal or oral sex in the past $6 \mathrm{mo}$ ); most were $\geqslant 35$ years of age (58\%), African-American (58\%) or Latino (35\%), had not completed high school (56\%), and had psychiatric diagnoses of schizophrenia or schizoaffective disorder $(57 \%)$ or major or bipolar depression (27\%). For sexually active men, follow up was $100 \%$ at 6 months and $95 \%$ at 18 months.

\section{Intervention}

52 men were allocated to the Sex, Games, and Videotapes (SexG) intervention, which involved 15 interactive sessions (2 $\mathrm{d} / \mathrm{wk}$ for $8 \mathrm{wks}$ ) facilitated by a mental health professional and a paraprofessional. Activities included storytelling, competitive games, acting scenes from their daily lives, and practising the decision making skills required for consistent condom use. 45 men were allocated to the control intervention which involved 2 sessions on HIV infection and other sexually transmitted diseases and basic instruction on condom use.

\section{Main outcome measures}

Main outcome was a sexual risk index (the Vaginal Episode Equivalent [VEE]) based on a semistructured interview given by interviewers blinded to the participant's group assignment. The index assigned scores for each unprotected anal, vaginal, and oral sexual episode. Other outcomes included episodes of sex, episodes with condom use, and high risk episodes (multiple partners and unprotected anal or vaginal sex).

\begin{abstract}
Main results
Intention to treat analysis focused on the subgroup of men who were sexually active at baseline $(\mathrm{n}=33$ in the SexG group and $\mathrm{n}=26$ in the control group). At 6 months, sexually active men in the SexG group had a lower mean VEE index than sexually active men in the control group (1.0 $v 3.1, \mathrm{p}=0.01)$. Although the total number of episodes of sex were similar for the 2 groups (261 v 247), the SexG group had a higher proportion of episodes with condom use than the control group (73\% v 52\%, $\mathrm{p}=0.04)$, and a lower proportion of high risk episodes $(24 \% \mathrm{v}$ $58 \%, \mathrm{p}=0.01)$. The between group difference in mean VEE scores decreased over time from 2.1 at 6 months to 1.6 at 18 months.

Among the 38 men who were not sexually active at baseline $(n=19$ in each group), the SexG group did not differ from the control group for number who were sexually active (11 $v 9)$ or had unprotected sex $(5 v 6)$ at 18 months.
\end{abstract}

\section{Conclusion}

A skills training intervention reduced high risk sexual behaviours in sexually active homeless men who had mental illness.

Sources of funding: US National Institute of Mental Health and US Centers for Disease Control and Prevention.

For correspondence: Dr E Susser, Columbia University: New York State Psychiatric Institute, $722 \mathrm{~W}$ 168th Street, Box 24, New York, NY 10032, USA. Fax +1 2127959768

\section{Commentary}

This small study by Susser et al raises interesting questions about a high risk, vulnerable, and sometimes sexually exploited group. The study clearly has implications for the community at large. As with other attempts to change the risk posed by such a mobile, stigmatised clientele, there is a paucity of good research in this area of mental and public health nursing. Monitoring of actual health outcomes to confirm self reported behaviour is important for studies of the effects of preventive health education. The study could have been strengthened by acquiring relevant medical histories of treatment for sexually transmitted diseases including HIV; documentation of appointments at relevant clinics; or evidence to support increased condom use. The study used a summary measure of sexual activity (VEE index) which relied on self report; further information about the validity and reliability of this measure may be available in other work by the authors. ${ }^{1}$

It is surprising that, in this study, the distinction between risk reduction and prevention is blurred. That is, men who were currently sexually active (risk reduction group) and men who were not (prevention group) received the same intervention. Some may argue that men who were not sexually active might learn risk behaviours through group discussions with sexually active men.

At the heart of this work is a reminder to practising professionals of the importance of sustained small group work which uses sound techniques of social skills training and group therapy. It should be applauded that psychiatric nurses can, and often do, challenge such socially taboo areas with skill, compassion, and humour.

The findings of this study could give outreach workers a strategy for engaging this difficult client group, but only after they have been validated by a wider multicentre study with a clear demarcation between prevention and risk reduction.

Steve Holder, RMN Community Psychiatric Nurse South West Outreach Team Edinburgh Healthcare NHS Trust Edinburgh, UK

1 Susser E, Desvarieux M, Wittkowski K. A risk index method for reporting sexual risk behayior for HIV. Am J Public Health; in press. 